\title{
A Simple Tool for Infection Prevention and Control Practice Surveillance: Development and use for Designing Feasible Programs in Egyptian Hospitals
}

\author{
Fatma Abdelaziz Amer ${ }^{1 *}$, Ahmed Ashraf Wegdan² and Heba AliMohtady ${ }^{1,3}$ \\ ${ }^{I}$ Medical Microbiology and Immunology Department, Zagazig Faculty of Medicine, \\ Zagazig, Egypt \\ ${ }^{2}$ Medical Microbiology and Immunology Department, Elfayoum \\ Faculty of Medicine, Elfayoum, Egypt \\ ${ }^{3}$ Fakeeh College for Medical Sciences, Kingdom of Saudi Arabia \\ *Corresponding author
}

\section{A B S T R A C T}

\section{Keywords \\ Infection prevention and control, Egypt, \\ Practice \\ surveillance, \\ Check-list, \\ Surveillance tool, \\ Middle- income \\ countries, Low- \\ resource setting \\ Article Info \\ Accepted: \\ 07 February 2019 \\ Available Online: \\ 10 March 2019}

In developing countries, it may be difficult to survey for nosocomial infections (NI). Moreover, a structure- based rather than internationally recommended infection prevention and control (IPC) programs are more applicable. IPC practice surveillance could be an additional or substituent survey. The current work describes the development of a measurable and updated tool for surveillance of IPC practices which we called "Ranked IPC Audit Check- Lists (RIPCACL)" and to evaluate their use for tailoring IPC programs in two hospitals in Egypt. The development addressed; first IPC procedures and second the needed resources. The check- lists were rendered measurable through a simple scoring system. They were used twice in two university hospitals. The first time was to capture a base- line IPC practice level, so IPC programs were tailored. The second was for monitoring and evaluation. The first implementation determined the practice levels in the two hospitals and identified the problematic areas. Further analyses of individual check list revealed the exact causes of trifling levels. Two IPC programs were tailored and implemented. Second implementation identified areas that could be upgraded and those which remained in a need for interventions. RIPCACL were convenient, flexible and easily applicable. They can be used in similar settings.

\section{Introduction}

IPC programs are essential for providing appropriate healthcare service and for promoting patient safety. Surveillance is the initial logical step of an IPC program. Surveillance data is needed to create, implement, monitor and evaluate IPC interventions, Pittet (2005). Surveillance of NI has been the gold standard for IPC programs. In developing countries and in lowresources settings, studies have shown that this surveillance is challenging, particularly at early stages of IPC implementation 
(Mahomed et al., 2017; Alp et al., 2011). Healthcare organizations may choose to include other surveillance to replace or supplement NI surveillance.

IPC practice surveillance which determines compliance with IPC measures can be an acceptable choice, Amer (2017). At the core of this surveillance approach is the checklist which is an elegant and simple tool. Checklists have been a big part of the significant success in the battle against NI (Abraham and Schwab, 2004; World Alliance for Patient Safety, 2018; the Joint Commission in collaboration with other international organizations, 2018). Nowadays, checklist protocols are provided by reputable international organizations, and postimplementation reports are available, predominantly, from industrialized countries. The checklists have proven to work best in establishments with high resources, supported by leaders who prioritize safety in daily care routines, Parand (2014). Because the situation is different in low- resource settings, the internationally- released checklists need to be modified (Amer et al., 1997a). An important novelty is to render them measurable, so the baseline situation, monitoring and evaluation statuses are translated into figures (Amer et al., 1997b) which support valid comparative analyses. Two decades ago, we successfully developed and implemented a simple tool which we called "Ranked Infection Control Audit Check- Lists (RICACL)" to design IC programs for the Cost Recovery for Health Project, in a pilot of 5 facilities in Egypt, Hassan (2008). In the early 2000s, at the request of John Snow Incorporation (JSI) and in partnership with the Egyptian Ministry of Health and Population, these checklists were adapted, upgraded and used successfully to design and implement IC program in a diverse set of facilities in Upper Egypt, John Snow Incorporation (2018). Currently, in 2018 great progress has been achieved in the area of IPC.
So, there is now a need to upgrade and update our previously developed RICACL and then to assess their value for designing appropriate IPC programs.

The current work describes the innovation process of the RICACL and their implementation for tailoring applicable IPC programs in two university hospitals in Egypt; Zagazig and El-Fayoum University Hospitals

\section{Materials and Methods}

RICACL for IPC practice surveillance were translated to the native language (Arabic). The updating process addressed two components. The first was the IPC procedures consistent with implementation of IPC program and the second was the resources required to carry-out the IPC procedures. To cope with the new terminology, RICACL has been termed Ranked Infection Prevention and Control Audit Check- Lists (RIPCRCLs).

The IPC procedures included both basics (handwashing, cleaning, disinfection, sterilization and isolation precautions) as well as departmental procedures for operation room (OR), intensive care unit (ICU), neonatal intensive care unit (NICU), hemodialysis unit (HU), laboratory (Lab), radiology department (RD), physiotherapy department (PD), laundry department (LD), dietary department (DD) and maintenance and engineering department (M/ED). Each checklist consisted of several core sections under which a series of relevant IPC elements were enumerated. The sections and elements were developed after reviewing and adapting a number of recently published guidelines such as CDC, World Health Organization (WHO) and others whenever possible.

Resources section encompassed all requirements of an effective, integrated IPC program across the healthcare continuum. 
They included: 1) human resources factors, i.e. training, staffing ratio in various services and departments and occupational health, 2) infrastructures elements including building environment, architectural designs, ventilation systems, number of handwashing facilities/hospital area and hospital incinerator, 3) equipment-related items including medical devices, smart accessories, bedside mobile monitors and others, existence of maintenance service and software packages, and 4) direct patient care factors, including bed occupancy, consumables and disposables, personal protective equipment, signs and taps of isolation, linen and bedclothes and decontamination equipment.

The scoring system used before (11), was utilized: 1) the score of an individual checklist was $100 \%$ distributed among core and the resource sections, 2) a positive "yes" (coded as 1) for each IPC/resource element indicated implementation/existence of that element. All other findings ("no", "not sure" or "sometimes") were coded as zero (Table 1). The grades of each IPC practice measured by an individual check- list or the grades of practice of the whole facility measured by all check- lists are converted into percentage score for each list or for the whole facility by simple mathematical calculation. Results were presented as tables or graphs to allow easy visual interpretation and comparison.

Check- lists were then reviewed by the infection control committee (ICC) of the two hospitals to evaluate their comprehensibility and to ensure accordance with services provided, patient population and environment with modification being made accordingly. Consistent with ICC's recommendations, the key measure or resource section scoring less than $50 \%$ was considered a problematic area.

Two (one- day) orientation workshops were carried out in each facility; the first was for the infection control team (ICT) and the second was for all the healthcare workers (HCW).

Check- lists were implemented twice. The first time was to capture a base- line IPC practice level and to determine area of problems. ICC members had access to collected data that supported tailoring IPC programs and subsequent implementation. The second implementation of the check- lists was after 6 months for monitoring and evaluation.

\section{Findings}

A compilation of 15 "RIPCACLs" were finalized for use in each hospital. The hand washing check- list contained 30 IPC/resource elements and was given $20 \%$ of the total score. Other check- lists; cleaning, disinfection, sterilization, isolation precautions, OR, ICU, NICU, HU, Lab, RD, $\mathrm{PD}, \mathrm{LD}, \mathrm{DD}$ and $\mathrm{M} / \mathrm{ED}$ contained the following IPC/resource elements and were given the percentage score shown between brackets; 73 (10\%), 60 (10\%), 70 (10\%), 60 (10\%), 45 (5\%), $30(5 \%), 30(5 \%), 50(5 \%)$, $50(5 \%), 30(3 \%), 20(2 \%), 40(4 \%), 40(4 \%)$, $20(2 \%)$, respectively.

The first use of the "RIPCACLs" identified an acceptable overall IPC practice score of $386 / 648$ (60\%) in Zagazig University hospital. Detailed analysis of individual practices revealed the followings to be areas for improvements; hand washing (10/30, $33.3 \%)$, isolation precautions $(20 / 60,33.3 \%)$, RD (10/30, 33.3\%), PD (5/20, 25\%), LD $(10 / 40,25 \%)$, DD $(10 / 40,25 \%)$ and M/ED $(5 / 20,25 \%)$. HU and Lab scored at the low acceptable levels (60\%, 52\% respectively). Other levels had a good score; cleaning; $82 \%$ disinfection; 83\%- sterilization; 86\%- OR; 88\%- ICU; 84\%- NICU; $84 \%$. 
In Elfayoum university hospitals, the first implementation of all check- lists identified acceptable overall IPC practice score of 354/648 (55\%). Detailed analysis of individual practices revealed the same areas identified in Zagazig University hospital to be areas for improvements (hand washing (10/30, 33.3\%), isolation precautions (20/60, $3.3 \%)$, RD (7/30, 23.3\%), PD (3/20, 15\%), LD (10/40, 25\%), DD (10/40, 25\%) and maintenance/engineering department (3/20, $15 \%$ ) in addition to the lab; 23/50, 46\%. HU scored at the low acceptable levels (25/50, $50 \%)$. Other levels had a good score; cleaning;55/73, 75\%- disinfection; 45/60, 75\%- sterilization; 56/70, 80\%- OR; 40/45, $88 \%$ - ICU; 25/30, 84\%- NICU; 22/30, 74\%.

Further analyses of the check- lists data attributed suboptimal scores in both hospitals to factors related to infrastructure, lack of adequate resources, insufficient human power, increased workload and deficiency in training

Based on previous results' two ICP programs were tailored for Zagazig and Elfayoum University hospitals. The programs consisted of prioritizing funds allocated to IPC, working to make all resources required for ICP practices available and providing educational (formal and on the job) and motivational programs. Alcohol rub procedure was emphasized and dispensers for handrubbing were distributed throughout the two hospitals to enhance compliance with hand hygiene procedures.

After six months the check- lists were implemented for a second time. In Zagazig University hospital, the overall IPC practice level was (441 point; 68.1\%). Significant changes were achieved in hand washing $(21 / 30,70 \%)$. Non- significant changes were encountered in cleaning; 62/72 (85\%)disinfection; 51/60 (85\%)- sterilization; 67/70
(95\%)- OR; $41 / 45$ (91\%)- ICU; 27.6/30 (92\%)- NICU; 28/30 (94\%)- HU; 35/50 (70\%)-lab; $33 / 50 \quad(65 \%)$. Isolation precautions; 21/60 (35\%)- RD; $10 / 30$ (34\%)PD; 7/20 (37\%)-LD 14/40 (35\%)- DD 16/40 (40) and maintenance/engineering department; 6/20 (30\%) remained areas for improvement.

In Elfayoum University hospital the overall IPC practice level was (365 point; 56.3\%). Significant changes were encountered as regards hand washing (20/30, 65\%). Nonsignificant changes were determined in cleaning; 62/73 (85\%)- disinfection; 45/60 (90\%)- sterilization; 63/70 (90\%)- OR; 41/45 (90\%)- ICU; 27/30 (90\%)- NICU; 24/30 (80\%)- HU; 30/50 (60\%)- lab; $27.5 / 50$ (55\%). Isolation precautions; 24/40 (40\%)- RD; $12 / 30$ (40\%)- PD; 5/20 (25\%)- LD; 12/30 (30\%)- DD; 16/40 (40\%) and M/ED; 5/20 $(25 \%)$ remained areas for improvements.

Figure 1 illustrates base line and postintervention levels of overall IPC practice in the two university hospitals. Figure 2 and 3 show base- line and post intervention IPC practice levels of individual practices in Zagazig and Elfayoum University Hospital, respectively. Significant improvements was achieved in hand washing $(\mathrm{P}<0.001)$

\section{Results and Discussion}

After about three decades of NI surveillance, it still remains an important problem for healthcare facilities in low- resource settings (Alp et al., 2011; Nejad et al., 2011; Samuel et al., 2010; Lowman, 2016). Many hospitals in developing countries concluded that implementation of NI surveillance is challenged by deficient human resources leading to increased work load, inadequate oversight due to deficiencies in training, substandard operating procedure because surveillance is not linked to routine data 
collection and lack of standardization on the diagnosis of hospital- acquired infections (HAI). Usually, commitment by hospital staff and hospital administration is poor (Saloojee and Steenh off, 2001). Despite the increased awareness of IPC aims and objectives, NI is considered a stigma, a finding that may be attributed to strong cultural or political bias (Currie et al., 2018). Poor laboratory backup (Gurley et al., 2010) and deficient funding are obstacles to implement NI surveillance.

Improperly designed information technology and the incomplete medical records add to the challenge (Zegers et al., 2011). So far medical records available in most healthcare facilities in developing countries are the traditional paper-based which are gathered and kept by healthcare employees. Retrieving data can be time-consuming, difficult or unreachable, O'Connor (2015); Modi (2018).

IPC practice surveillance is a convenient option, particularly at early stages of IPC implementation and it paves the way for surveying NI. IPC practice measures compliance with general basic and departmental IPC procedures. It does not require Microbiology laboratory aid. It can be on- site conducted through direct observation and interviews without need for reference to patient records or for follow-up after discharge. It is more acceptable by HCWs and hospital administration, especially if convinced that the surveillance identifies issues before evolving into NIs with subsequent stigmatization.

Our previous work demonstrated that IC practice surveillance is the optimum for healthcare facilities providing outpatient service (Amer et al., 1997b) because it does not necessitate post-discharge surveillance methodologies that are impeded by the need for allocation of extra cost, time and training for both data collectors and patients. Finally, it is a common sense that upgrading practices should decrease NI.

In this study, we used checklists as the surveillance tool. Since their first implementation in the aviation profession in 1935, Code7700 (2018), checklists are widely used in many areas. The checklist first gained attention in medical practice when Peter Pronovost, Laurence (2009), embraced its use to improve outcomes in intensive care settings in Michigan hospitals. His experience published in the New England Journal of Medicine (Pronovost et al., 2006), is now known as the landmark Keystone Initiative and it gained national attention when The New Yorker magazine published "The Checklist" in 2007, Gawande (2007).

RIPCACLs were developed in Arabic to allow their easily use. Since IPC practice includes both basic and specific departmental procedures, RIPCACLs were developed for both. They were designed so that IPC personnel will not need to rely on their memory for items to be checked. Resource identification provides rationale for the demand of financial support and other essential needs crucial for the IPC program and its success. This includes the availability of water, soap or an equivalent (e.g. alcohol rub), before emphasizing the importance of hand sanitation.

Ranked IC audit checklists provided a flexible tool for assessing the exact need of facilities with different resources, environment, patient population and services provided. Their scoring system contributed also to the flexibility. It is to be emphasized that on distribution of scores, the practice having the greatest positive impact on IPC in a particular setting should be considered whether for an individual checklist or for a compilation of checklists. In the current work, the hand washing checklist was given the highest score 
because improved adherence to hand hygiene is extensively accredited to be the greatest significant way of decreasing infections in health care facilities (Saito et al., 2018).

Table.1 An example for an ICP ranked audit check- list. The number of core sections/ICP elements varies between various practices, consequently, the percentage score. In each case the sum of the ICP elements is converted into score as shown

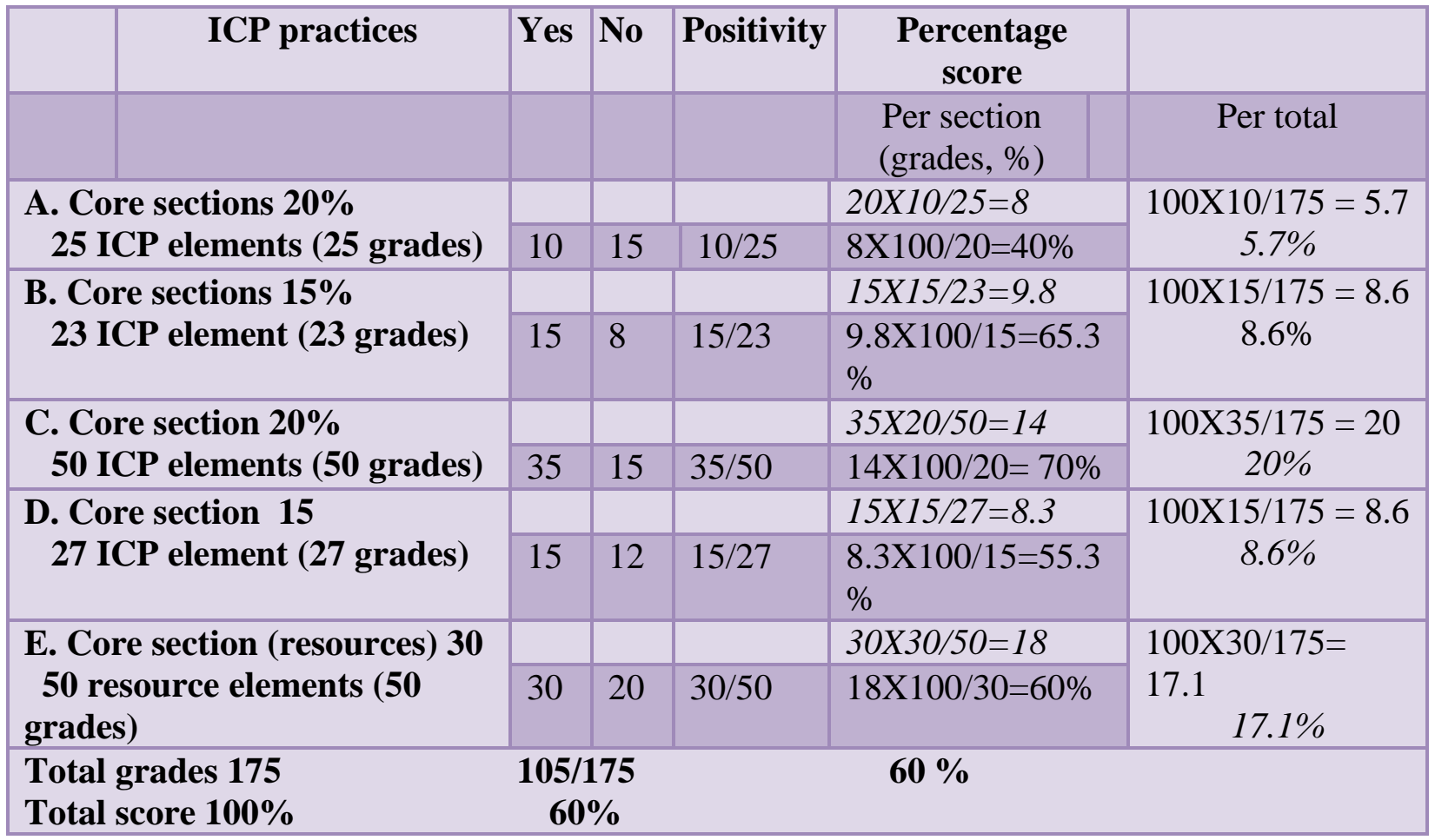

Fig.1 Base line and post- intervention levels of overall IPC practice in the two university hospitals

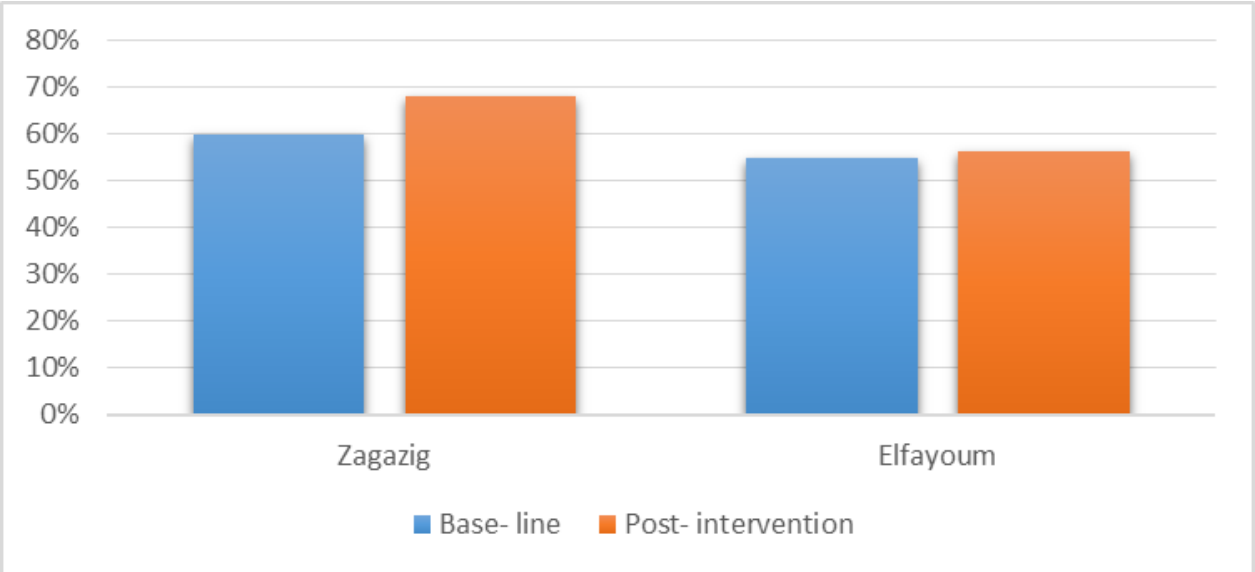


Fig.2 Base- line and post intervention IPC practice levels of individual practices in Zagazig University Hospital. Significant improvements was achieved in handwashing $(P<0.001)$

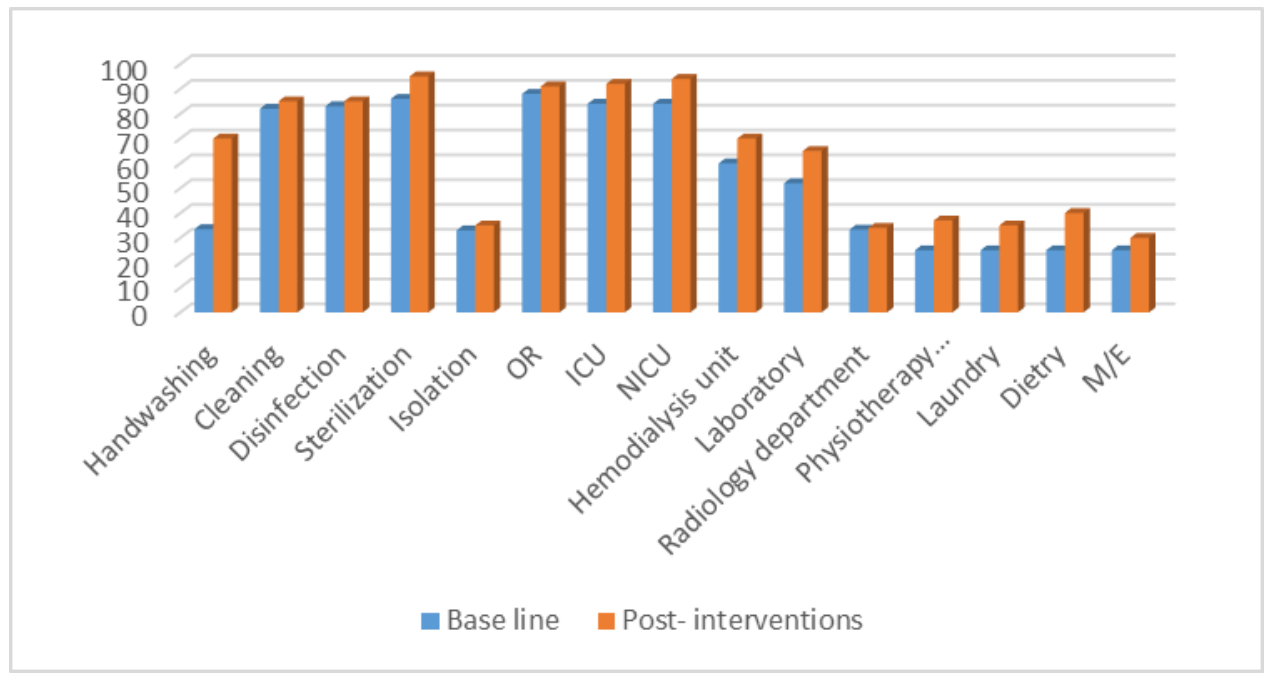

Fig.3 Base- line and post intervention IPC practice levels of individual practices in Elfayoum University Hospital. Significant improvements was achieved in handwashing $(P<0.001)$

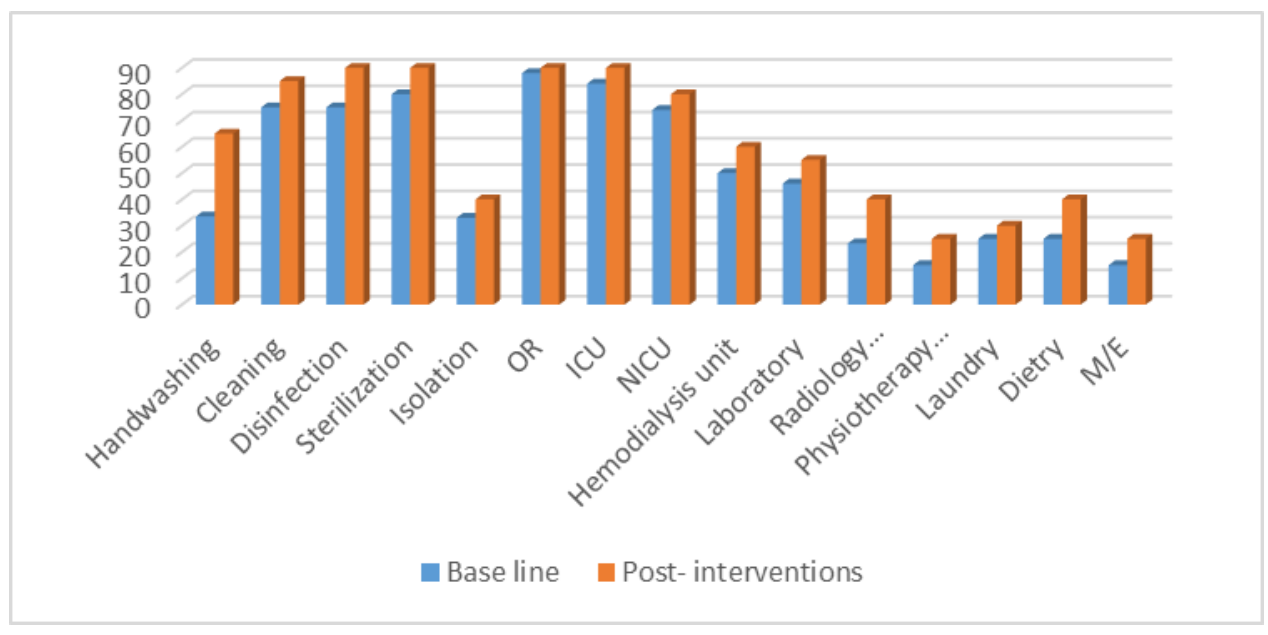

Levels of IPC practice measured by $1 / 0$ score and expressed as absolute numbers are neutral and means done/not-done. In this respect, they resemble levels of performance measured for improving the quality of patient care, Lighter (2015). Their importance is related to comparative purposes between starting (base line) and post IPC program implementation. Moreover, selecting less than half the percentage to indicate problematic area is a relative issue. Lower or higher values may be chosen based on individual institution. In this respect RIPCACLs differ from checklists used by accreditation organizations, e. g., the Joint Commission for Accreditation of Healthcare Institutions (JCAHI) and Saudi Central Board for Accreditation of Healthcare Institutions (CBAHI). These establishments are authorized to assess and grant accreditation to healthcare facilities through field visits conducted by professional surveyors. The certificates are granted if the 
facility complies with a package of quality and patient safety standards and a predetermined score has to be achieved, the Joint Commission (2019); CBAHI (2019).

RIPCACLs provided guidelines for tailoring feasible IPC programs. Considering the context of developing countries and settings of limited resources, it is difficult for a program designed to meet preconceived set of standards to solve problems (Amer et al., 1997a). Furthermore, individual peculiarities can make it hard to propose the same IPC program for all facilities.

Through their development and implementation, RIPCACLs were a learning experience. Information contained in their bodies pertained to detailed IPC procedures which should be known by all HCW. Discussion which took place then to finalize the checklist and coordinated by IPC consultants (expert power), (Reid and Kawash, 2017) allowed the supply of more information (information power).

Supply of information by experts is essential and achieves the best compliance. Finally, data collection activities required frequent reading to prevent necessary IPC step from ever being forgotten. Reading is another training method in addition to being an experimental learning experience (Sahatcija et al., 2017).

In conclusion, RIPCACLs are supportive resource that are adjunct to or may replace NI surveillance particularly in poor- resources settings. They provide help to infection preventionists in preliminary assessment of IPC practices, performing ongoing data analysis, creating structured IPC programs and allowing for monitoring and evaluation that permit for patient improvement opportunities.

\section{References}

Abraham, P., and Schwab' A.P. 2004. Ethics in the Practice of Surgery. AMAJ Ethics. 6:456-458.

Alp, E., Leblebicioglu, L., Doganay, M., et al., 2011. Infection control practice in countries with limited resources. Ann Clin. Microbiol. Antimicrob. 10: 36.

Amer, F. 2017. Hospital Infection Control, In Program Organization. $3^{\text {rd }}$ ed. Noor Publishing. P: 178-179.

Amer, F.A., Raaft, A., and Abdelfatah, S. 1997. Adaptation of JCAHO standards for surveillance, prevention and control of infection in a healthcare facility in Egypt. The IFIC Global Network Conference on Safe and Unsafe Methods of Infection Control. Cambridge, United Kingdom.

Amer, F.A., Raaft, A., and Abdelfatah, S. 1997. Infection control (IC) program implementation using ranked IC audit check-lists: an Egyptian experience. The IFIC Global Network Conference on Safe and Unsafe Methods of Infection Control. Cambridge, United Kingdom.

CBAHI (Saudi Central Board for Accreditation of Healthcare Institutions). 2019. Available from: https://portal.cbahi.gov.sa/english/home

Code7700. 2018. The early days of checklists in aviation. In: Checklist Flows- Normal Procedures. Available from: http://code7700.com/checklist_flows.ht $\mathrm{m}$.

Currie, K., Melone, L., Stewart, S., et al., 2018. Understanding the patient experience of health care-associated infection: A qualitative systematic review. Am J Infect Control.46:936942.

Gawande, A. 2018. The Checklist. Available from:

https://www.newyorker.com/magazine/ 
2007/12/10/the-checklist.

Gurley, E. S., Zaman, R. U., Sultana, R., et al., 2010. Rates of hospital-acquired respiratory illness in Bangladeshi tertiary care hospitals: results from a low-cost pilot surveillance strategy. Clin Infect Dis. 50: 1084-1090.

Hassan, M. K. 2008. Cost Recovery for Health Project (USAID Project no.: 263-0170), Cairo, Egypt, In Corporate Governance in Less Developed and Emerging Economies (Tsamenyi $\mathrm{M}$. and Uddin S., Eds) pp 431. Emerald Group Publishing, Amazone.com

John Snow Incorporation (JSI), Cairo, Egypt. 2018. Healthy Mother/Healthy Child Project Completion Report. Available from: https://www.jsi.com/JSIInternet/Resour ces/publication/display.cfm?txtGeoArea $=\mathrm{I}$

NTL\&id=4313\&thisSection=Resources.

Laurence, J. 2009. Peter Pronovost: champion of checklists in critical care. Lancet 2009; 374.

Lighter, D. E. 2015. Quality improvement; How (and why) do quality improvement professionals measure performance? Int J PediatrAdolesc Med. 2: 7-11.

Lowman, W. 2016. Active surveillance of hospital-acquired infections in South Africa: implementation, impact and challenges. S Afr Med J. 106:489-493.

Mahomed, S., Mahomed, O., Sturm, A. W., et al., 2017. Challenges with surveillance of healthcare- associated infections in intensive care units in South Africa. Crit Care Res Pract. 7296317.

Modi, A. 2018. 5 top disadvantages of manual medical records. Available from: https://www.linkedin.com/pulse/5-topdisadvantages-manual-medical-recordsarti-modi/.

Nejad, S. B., Allegranzi, B., Syed, S. B., et al., 2011. Health-care-associated infection in Africa: a systematic review.
Bull WHO. 89:757-765.

O'Connor, S. Why your paper-based system is slowing your practice down. 2015. https://www.adsc.com/blog/why-yourpaper-based-system-is-slowing-yourpractice-down. Last accessed October 1st, 2018.

Parand, A., Dopson, S., Renz, A., et al., 2014. The role of hospital managers in quality and patient safety: a systematic review. BMJ. Retrieved from http://dx.doi.org/10.1136/bmjopen2014-005055. Last accessed September 28th, 2018

Pittet, D. 2005. Infection control and quality health care in the new millennium. Am J Infect Control.33:258-267,

Pronovost, P., Needham, D., Berenholtz, S., et al., 2006. An intervention to decrease catheter-related bloodstream infections in the ICU. N Engl J Med. 355:26.

Reid, L. F., and Kawash, J. 2017. Let's talk about power: How teacher use of power shapes relationships and learning. Papers on Postsecondary Learning and Teaching: Proceedings of the University of Calgary Conference on Learning and Teaching, 2, 34-41.

Sahatcija, R., Ora, A., and Ferhataj, A. 2017. The Impact of the thinking style on teaching methods and academic achievement. ESJ. 13: 1857 - 7881.

Saito, H., Kilpatrick, C. andPittet, D. 2018. The World Health Organization SAVE LIVES: Clean Your Hands Campaign targets sepsis in health care. Intensive Care Med. 44: 499.

Saloojee, H., and Steenhoff, A. 2001. The health professional's role in preventing nosocomial infections. Postgrad Med J. 77:16-19.

Samuel, S., Kayode, O., Musa, I., et al., 2010. Nosocomial infections and the challenges of control in developing countries. Afr J Cln Exper Microbiol. 11:102-110. 
The Joint Commission in collaboration with other international organizations. 2018. Measuring Hand Hygiene Adherence: Overcoming the Challenges. Available from: https://www.jointcommission.org/ assets/1/18/hh_monograph.pdf

The Joint Commission. What is Accreditation? 2019. Available from: https://www.jointcommission.org/accre ditation/accreditation_main.aspx

World Alliance for Patient Safety.2018. WHO
Surgical Safety Checklist and Implementation Manual. Available from: http://www.who.int/patientsafety/ safesurgery/ss_checklist/en/).

Zegers, M., De Bruijne, M.C., Spreeuwenberg, P., et al., 2011. Quality of patient record keeping: an indicator of the quality of care? BMJ QualSaf. 20:314-318.

\section{How to cite this article:}

Fatma Abdelaziz Amer, Ahmed Ashraf Wegdan and Heba AliMohtady. 2019. A Simple Tool for Infection Prevention and Control Practice Surveillance: Development and use for Designing Feasible Programs in Egyptian Hospitals. Int.J.Curr.Microbiol.App.Sci. 8(03): 453-462. doi: https://doi.org/10.20546/ijcmas.2019.803.057 